\title{
ORTHODOX SEMIRINGS AND RINGS
}

\author{
JOHN ZELEZNIKOW
}

(Received 3 April 1979)

Communicated by T. E. Hall

\begin{abstract}
We show that in a regular ring $(R,+, \cdot)$, with idempotent set $E$, the following conditions are equivalent:

(i) $\forall e, f \in E,(e \cdot f=0 \Rightarrow f \cdot e=0)$.

(ii) $(R, \cdot)$ is orthodox.

(iii) $(R, \cdot)$ is a semilattice of groups.

These and other conditions are also considered for regular semigroups, and for semirings $(S,+, \cdot)$, in which $(S,+)$ is an inverse semigroup. Examples are given to show that they are not equivalent in these cases.
\end{abstract}

1980 Mathematics subject classification (Amer. Math. Soc.): primary 16 A 78; secondary 20 M 25.

\section{Preliminaries}

Result 1. (Chaptal (1966), Proposition 1.) For a ring $(R,+, \cdot)$, the following conditions are equivalent.

(i) $(R, \cdot)$ is a union of groups.

(ii) $(R, \cdot)$ is an inverse semigroup.

(iii) $(R, \cdot)$ is a semilattice of groups.

Definirion 2. A triple $(S,+, \cdot)$ is a semiring if $S$ is a set, and,$+ \cdot$ are binary operations satisfying

(i) $(S,+)$ is a semigroup,

(ii) $(S, \cdot)$ is a semigroup,

(iii) $a \cdot(b+c)=a \cdot b+a \cdot c,(a+b) \cdot c=a \cdot c+b \cdot c$, for all $a, b, c \in S$.

For any $a, b \in S$, we frequently denote $a \cdot b$ by $a b$.

Definition 3. An element $a \in S$ is an additive zero if $x+a=a+x=x$ for all $x \in S$. An element $b \in S$ is a multiplicative zero if $x \cdot b=b \cdot x=b$ for all $x \in S$. 
If $(S,+, \cdot)$ does not have an element which is both an additive and a multiplicative zero, form $S^{0}=S \cup\{0\}$, where $x+0=0+x=x, x \cdot 0=0 \cdot x=0$ for all $x \in S$. We shall henceforth assume $(S,+, \cdot)$ has an element 0 which is both an additive and a multiplicative zero.

Definition 4. In a semiring $(S,+, \cdot)$, we put $E^{[+]}=\{x \in S: x+x=x\}$ and $E^{[1]}=\{e \in S: e \cdot e=e\}$ and for each $x \in S$ we define $V^{[+}(x)=\{a \in S: x+a+x=x$ and $a+x+a=a\}$ and $V^{1}(x)=\{b \in S: x \cdot b \cdot x=x$ and $b \cdot x \cdot x=b\}$. We denote by $x^{\prime}\left[\right.$ respectively $\left.x^{*}\right]$ an element chosen from $V^{[+1}(x)$ [respectively $\left.V^{[1}(x)\right]$, when this set is nonempty.

A semiring $(S,+, \cdot)$ is said to be an additively inverse semiring if $(S,+)$ is an inverse semigroup.

Definition 5. A semigroup $(S, \cdot)$ is orthodox if it is regular and $E=\{e \in S: e \cdot e=e\}$ is a subsemigroup of $S$.

We shall require the following results.

Result 6. (Grillet (1970), Lemma 2(i).) For any semiring $(S,+, \cdot)$, the set $E^{[+1}$ is an ideal of $(S, \cdot)$.

Result 7. (Karvellas (1974), Theorem 3(ii) and Theorem 7.) Take any additively inverse semiring $(S,+, \cdot)$.

(i) For all $x, y \in S,(x \cdot y)^{\prime}=x^{\prime} \cdot y=x \cdot y^{\prime}$ and $x^{\prime} \cdot y^{\prime}=x \cdot y$.

(ii) If $a \in a S \cap S a$ for all $a \in S$ then $S$ is additively commutative (and hence additively a semilattice of commutative groups).

We use the definitions and notation of Clifford and Preston (1961).

\section{Orthodox semirings}

LEMMA 8. Take any regular semigroup $(S, \cdot)$ with zero 0 and set of idempotents $E$. Then the following conditions are equivalent.

(i) $\forall e \in E, \forall x \in S,(e \cdot x=0 \Rightarrow x \cdot e=0)$.

(ii) $\forall n \in \mathbf{N}, \forall x \in S,\left(x^{n}=0 \Rightarrow x=0\right)$.

(iii) $\forall x \in S,\left(x^{2}=0 \Rightarrow x=0\right)$.

(iv) $\forall x, y \in S,(x \cdot y=0 \Rightarrow y \cdot x=0)$.

Proof: (i) $\Rightarrow$ (ii). Take any $x \in S$ with $x^{n}=0$ for some $n>1$. Take any inverse $x^{*}$ of $x$ in $S$. Then $x^{*} x^{n}=0$ and so $\left(x^{*} x\right) x^{n-1}=0$. But $x^{*} x \in E$ and thus $x^{n-1}\left(x^{*} x\right)=0$. 
Hence $x^{n-2}\left(x \cdot x^{*} \cdot x\right)=0$, i.e. $x^{n-1}=0$; and continuing this process, we have $x=0$.

(iii) $\Rightarrow$ (iv). Take any $x, y \in S$ with $x y=0$. Then $(y x)^{2}=y(x y) x=y \cdot 0 \cdot x=0$ and thus $y x=0$. So (i), (ii), (iii), (iv) are equivalent.

THEOREM 9. Let $(S,+, \cdot)$ be any additively inverse semiring in which $(S, \cdot)$ is regular. The the following conditions are equivalent.

(i) $\forall e, f \in E^{[]},(e \cdot f=0 \Rightarrow f \cdot e=0)$.

(ii) $\forall e \in E^{[]}, \forall x \in S,(e \cdot x=0 \Rightarrow x \cdot e=0)$.

(iii) $\forall n \in \mathbf{N}, \forall x \in S,\left(x^{n}=0 \Rightarrow x=0\right)$.

(iv) $\forall x \in S,\left(x^{2}=0 \Rightarrow x=0\right)$.

(v) $\forall x, y \in S,(x \cdot y=0 \Rightarrow y \cdot x=0)$.

Further, each is implied by

(vi) $(S, \cdot)$ is orthodox.

ProOF: (i) $\Rightarrow$ (ii). Take $e \in E^{[]}, x \in S$, with $e \cdot x=0$. Then

$$
\begin{aligned}
\left(e+(x e)^{\prime}\right)^{2} & =e\left(e+(x e)^{\prime}\right)+(x e)^{\prime}\left(e+(x e)^{\prime}\right) \\
& =e \cdot e+(e x e)^{\prime}+(x e e)^{\prime}+x e x e \\
& =e+0^{\prime}+(x e)^{\prime}+0 \\
& =e+(x e)^{\prime} .
\end{aligned}
$$

Thus $e+(x e)^{\prime} \in E^{[\cdot]}$. Now $\left(e+(x e)^{\prime}\right)\left(x x^{*}\right)=e x x^{*}+\left(x e x x^{*}\right)^{\prime}=0+0^{\prime}=0$. But $e+(x e)^{\prime}, x x^{*} \in E^{[]}$, so $\left(x x^{*}\right)\left(e+(x e)^{\prime}\right)=0$ and thus $x x^{*} e+\left(x x^{*} x e\right)^{\prime}=0$. Hence $x x^{*} e+(x e)^{\prime}=0$; and so $x x^{*} e+(x e)^{\prime}+x x^{*} e=x x^{*} e$, and $(x e)^{\prime}+x x^{*} e+(x e)^{\prime}=(x e)^{\prime}$. Since $(S,+)$ is inverse, $x x^{*} e=x e$. Now $e x x^{*}=0$ and thus $x e=x x^{*} e=0$. Hence by Lemma 8 , we have that (i), (ii), (iii), (iv), (v) are equivalent.

(vi) $\Rightarrow$ (i). Take $e, f \in E^{[\cdot]}$ with $e \cdot f=0$. Since $f \cdot e \in E^{[\cdot]}$,

$$
(f e)=(f e)^{2}=f(e f) e=f \cdot 0 \cdot e=0 .
$$

EXAMPLE 10. In an arbitrary regular semigroup $(S, \cdot)$, condition (i) of Theorem 9 does not imply condition (ii), and $(S, \cdot)$ being orthodox does not imply condition (ii). To see this, we may take any Brandt semigroup $S=\mathscr{M}^{\circ}(G, I, I, \Delta)$ in which $|I| \geqslant 2$.

ExAmple 11. Let $(S,+, \cdot)$ be a regular ring in which $(S, \cdot)$ is not orthodox. Put $T=S \cup\{a\}$, where $a \notin S$ and define $s+a=a+s=s, a+a=a, s \cdot a=a \cdot s=a$ $=a \cdot a$, for all $s \in S$. Then $\left(T,+,^{\cdot}\right)$ is a semiring in which $(T,+)$ is inverse, $(T, \cdot)$ is regular, and $a$ is the additive and multiplicative zero of $T$. Hence $(T,+, \cdot)$ satisfies condition (i) of Theorem 9, but is not orthodox. 


\section{Orthodox rings}

DefinITION 12. A semigroup $(S, \cdot)$ is a ring-semigroup if there exists a binary operation + on $S$ such that $(S,+, \cdot)$ is a ring.

THEOREM 13. In a regular ring-semigroup $(S, \cdot)$, the following are equivalent.

(i) $(S, \cdot)$ is orthodox.

(ii) $\forall e, f \in E,(e \cdot f=0 \Rightarrow f \cdot e=0)$.

(iii) $\forall e \in E, \forall x \in S,(e \cdot x=0 \Rightarrow x \cdot e=0)$.

(iv) $\forall n \in \mathbf{N}, \forall x \in S,\left(x^{n}=0 \Rightarrow x=0\right)$.

(v) $\forall x \in S,\left(x^{2}=0 \Rightarrow x=0\right)$.

(vi) $\forall x, y \in S,(x \cdot y=0 \Rightarrow y \cdot x=0)$.

(vii) $(\mathrm{S}, \cdot)$ is inverse.

Proof. (vi) $\Rightarrow$ (vii). Take any $e, f \in E$. Then $e(f-e f)=0$ and $(f-f e) e=0$. So $(f-e f) e=0$ and $e(f-f e)=0$. Thus $f e=e f e=e f$. Since any inverse semigroup is orthodox, the theorem now follows from Theorem 9.

The above theorem does not hold if $(S,+, \cdot)$ is a semiring in which $(S,+)$ is inverse [orthodox] and $(S, \cdot)$ is orthodox [inverse], as is shown by the following examples.

EXAMPLE 14. (i) Let $(S,+)$ be a semilattice with $|S| \geqslant 2$ and define $x \cdot y=x$ for all $x, y \in S$. Then $x \cdot(y+z)=x$ and $x \cdot y+x \cdot z=x+x=x$. Also $(x+y) \cdot z=x+y$ and $x \cdot z+y \cdot z=x+y$. So $(S,+, \cdot)$ is a semiring in which $(S,+)$ is inverse and $(S, \cdot)$ is othodox (in fact a left zero band) but not inverse.

(ii) Let $(S \backslash\{0\}, \cdot)$ be a group and define $x+y=x$ for all $x, y \in S$. Then $x \cdot(y+z)=x \cdot y$ and $x \cdot y+x \cdot z=x \cdot y$. Also $(x+y) \cdot z=x \cdot z$ and $x \cdot z+y \cdot z=x \cdot z$. Hence $(S,+, \cdot)$ is a semiring in which this time $(S, \cdot)$ is inverse and $(S,+)$ is orthodox (in fact a left zero band) but not inverse.

REMARK 15. From Result 1 and Theorem 13, the following are equivalent for a regular ring-semigroup.

(i) $\forall e, f \in E,(e \cdot f=0 \Rightarrow f \cdot e=0)$.

(ii) $(S, \cdot)$ is orthodox.

(iii) $(S, \cdot)$ is inverse.

(iv) $(S, \cdot)$ is a semilattice of groups.

Since any ring $(S,+, \cdot)$ satisfying (iv) is regular and has no nonzero nilpotent elements, by Kovacs (1956), Theorem 2, the conditions (i)-(iv) are also equivalent to the condition

(v) $R$ is a subdirect sum of division rings.

From Result 1, for any ring $(R,+, \cdot)$, if $(R, \cdot)$ is a union of groups then $(R, \cdot)$ is a semilattice of groups. In Example 14(i), the semiring $(S,+, \cdot)$ has $(S,+)$ a semilattice, 
and $(S, \cdot)$ both orthodox and a union of groups. However, $(S, \cdot)$ is not a semilattice of groups.

\section{References}

N. Chaptal (1966), 'Anneaux dont le demi-groupe multiplicatif est inverse', C. R. Acad. Sci. Paris Sér. A-B 262, 274277.

A. H. Clifford and G. B. Preston (1961), The algebraic theory of semigroups, Vol. I (American Mathematical Society. Mathematical Surveys 7).

M. O. P. Grillet (1970), 'Subdivision rings of a semiring', Fund Math. 67, 67-74.

P. H. Karvellas (1974), 'Inversive semirings', J. Austral. Math. Soc. 18, 277-287.

L. Kovacs (1956), 'A note on regular rings', Publ. Math. Debrecen 4, 465-468.

Department of Mathematics

Monash University

Clayton, Victoria 3168

Australia

Author's current address:

Department of Mathematical Sciences

Northern Illinois University

De Kalb, Illinois 60115

U.S.A. 